\title{
Aux origines de la francophonie
}

La notion de « langue de civilisation »

Javier Suso López et Maria Eugenia Fernández Fraile

\section{(2) OpenEdition}

Journals

Édition électronique

URL : https://journals.openedition.org/dhfles/107

DOI : $10.4000 /$ dhfles. 107

ISSN : 2221-4038

Éditeur

Société Internationale pour l'Histoire du Français Langue Étrangère ou Seconde

Édition imprimée

Date de publication : 1 janvier 2008

Pagination : 77-100

ISSN : 0992-7654

Référence électronique

Javier Suso López et Maria Eugenia Fernández Fraile, «Aux origines de la francophonie », Documents pour l'histoire du français langue étrangère ou seconde [En ligne], 40/41 | 2008, mis en ligne le 20 novembre 2014, consulté le 27 mai 2021. URL : http://journals.openedition.org/dhfles/107 ; DOI : https://doi.org/10.4000/dhfles.107

Ce document a été généré automatiquement le 27 mai 2021

(c) SIHFLES 


\title{
Aux origines de la francophonie
}

\author{
La notion de « langue de civilisation » \\ Javier Suso López et Maria Eugenia Fernández Fraile
}

1 La francophonie est inséparable de la «langue »: la langue, à l'aide de laquelle le locuteur émet des messages destinés à être déchiffrés, est aussi un objet, livré à l'appréciation, à des jugements de valeur (Bourdieu, 2001). Notre objectif, au cours de cet article, est de poser la langue française comme lieu de réflexion au moment où la France se livrait pleinement à l'entreprise coloniale (période d'entre les deux guerres et années postérieures à la Seconde Guerre mondiale). L'intérêt pour démêler quelle construction idéologique accompagne la notion de langue française est d'autant plus pertinent que le terme «langue » a été associé à cette époque au terme "civilisation » pour obtenir l'expression «langue de civilisation». Quelle portée idéologique cette opération comporte-t-elle? Y aurait-il des langues de civilisation en opposition à d'autres langues qui ne posséderaient pas cette « valeur »?

2 Notre réflexion s'organise en deux volets qui se succèdent d'un point de vue chronologique : le premier veut montrer la genèse de cette expression, qui fait fortune dans les années 1920,30, 40 et 50, et les notions (multiples et complexes) auxquelles elle est rattachée, à travers les positionnements d'une série de linguistes du début du $\mathrm{XX}^{\mathrm{e}}$ siècle; une fois construite cette notion, et acceptée par la communauté des «intellectuels» de l'époque, elle est reprise pour appuyer une action politicolinguistique concrète, axée sur l'idée du français comme langue de civilisation (Unesco, Français élémentaire). Le tournant pris par la décolonisation écartera rapidement cette expression au profit de deux autres: le français «langue de culture » et «langue et civilisation françaises $»$.

\section{"Langue de civilisation » et pensée linguistique dans la première moitié du $X X^{e}$ siècle}

3 L'expression "langue de civilisation" doit être tout d'abord reliée au courant de pensée de la philosophie des Lumières, à une mentalité ouverte et généreuse, de confiance dans le progrès et de croyance dans la raison comme valeur commune des 
hommes. Comme le signale Émile Benveniste (1976), le mot civilisation a joué un rôle fondamental dans l'histoire de la pensée moderne, en tant que concept qui se construit à partir des «représentations qu'une collectivité humaine a d'elle-même, [d]es liens que les sociétés tissent entre elles, et [d]es jugements de valeur qu'elles ne manquent pas de porter les unes sur les autres" (Argaud 2006: 5). Disons aussi que le terme " civilisation » est très récent : il n'apparaît dans le Dictionnaire de l'Académie qu'à partir de l'édition de $1835^{1}$. Nous allons reprendre un bref texte d'Évelyne Argaud car il met en relief les implications idéologiques de cette notion :

Le terme civilisation [...] a longtemps désigné l'état d'un peuple qui a quitté sa condition primitive, qui s'est éloigné de l'état animal et sauvage par un processus moral, intellectuel et industriel, lui permettant de sortir de la barbarie et de s'améliorer, et, en tant que tel, il s'est chargé de connotations positives et valorisantes. La civilisation était une continuelle marche en avant, inséparable de l'idée de progrès. Même si les définitions des dictionnaires notent qu'on en est venu, peu à peu, à reconnaître l'existence de différentes modalités et de différents degrés dans la façon de réaliser la civilisation, cette foi dans la civilisation ne s'en est pas moins accompagnée, souvent, d'un ethnocentrisme qui condamnait le sauvage à une infériorité naturelle. Les différentes civilisations ne pouvaient qu'emprunter le chemin montré par les Européens, ou demeurer dans un état d'arriération. (Argaud $2006: 5-6$ )

4 C'est aussi le verbe civiliser qui reflète bien cette idéologie de la fin du XIX $\mathrm{X}^{\mathrm{e}}$ siècle et du début du XXe siècle, qui soutenait une hiérarchie des civilisations et des cultures (des langues et des races, de même), et qui constituait pour certains le bien-fondé de l'entreprise coloniale : "rendre policé et sociable un peuple qui vit à l'état sauvage » (Dictionnaire de l'Académie française, 8 éd., 1932-5 : 1243).

Sur cette composante essentielle vient se greffer une idéologie de la langue française, «mythe fondateur» de la pensée linguistique et stylistique, qui a tenu le français comme une langue (quasi) parfaite, et expression à la fois d'une culture d'exception. Ce discours sur la langue a été tellement répété depuis le XVII ${ }^{e}$ siècle qu'il s'est profondément ancré dans les esprits de toute la société française (et même francophone) : pour preuve ces lignes tirées d'un manuel scolaire (composé par M. Hénon), qui présente l'évolution de la langue française comme suit:

Les gouvernements étrangers commencèrent d'eux-mêmes dans la rédaction de leurs notes et de leurs documents à employer le français. On appréciait aussi partout les rares avantages de cet idiome pour ce qui est de la précision et de la clarté, deux qualités particulièrement précieuses pour les actes diplomatiques... En même temps le français était considéré comme la langue la plus élégante et la plus aristocratique de toute l'Europe... Sa beauté exerce une telle séduction qu'un grammairien allemand écrit qu'on doit parler français aux dames, et n'employer l'allemand que quand on est en colère et qu'on veut injurier. (1938: II, 23)

6 Le sommet des panégyriques sur la langue française se trouve en ce début du $\mathrm{XX}^{\mathrm{e}}$ siècle : «Puisqu'il faut le dire, elle est de toutes les langues, la seule qui ait une probité attachée à son génie. Sûre, sociale, raisonnable, ce n'est plus la langue française, c'est la langue humaine » (Rambaud 1911 : II, 346). Au-delà de ces manifestations adressées au grand public, voyons comment certains linguistes de l'époque se positionnent à l'égard de la question de la langue. Nous allons nous limiter pour des raisons d'espace à deux d'entre eux : Joseph Vendryes et Antoine Meillet.

7 La langue est-elle « une chose tout à fait extrahumaine, et en soi organisée, comme serait une végétation parasite répandue à la surface de notre espèce » (Petroff \& Engler 
2004: 134), comme l'affirment certains, ou bien la langue est-elle une institution humaine ? Voilà les termes de bien des débats linguistiques de la fin du XIX et du début $\mathrm{du} \mathrm{XX}^{\mathrm{e}}$ siècle, où l'on retrouve, bien que légèrement modifié, le débat cratyléen (naturalisme versus conventionnalisme). L'idée de la langue en tant qu'organisme vivant commence à perdre des défenseurs au profit du lien de la langue à la société et à l'homme. Cette évolution dans les prises de position marque également le développement de la notion de «langue de civilisation» vers une complexité de connotations qui vont lui être associées.

Que le langage (et donc toute langue particulière) soit le produit d'une société (sociolinguistique: Durkheim, Meillet, Vendryes, Lévy-Bruhl) ou le produit d'un cerveau humain en rapport étroit avec la vie psychologique (Henri Berr, Paul Janet) étaient des positions aisément réconciliables dans le fond: dans les deux cas, on marquait une forte dépendance de la langue à l'homme, et la langue n'était nullement quelque chose « en soi » (Saussure). Dans son ouvrage Le langage. Introduction linguistique à l'histoire (1921), qui fait partie d'une synthèse collective sur l'évolution de l'humanité, Joseph Vendryes révèle un état d'esprit largement partagé par les linguistes de l'époque. Pour lui, la langue n'est pas quelque chose d'indépendant des hommes : « il est faux de considérer le langage comme une entité idéale évoluant indépendamment des hommes et poursuivant ses fins propres » $(1921: 420)$. Vendryes marque un rapport complexe du langage à l'homme où physiologie, psychologie, société et histoire doivent être pris en compte: "le langage [...] est un acte physiologique en ce qu'il met en œuvre plusieurs organes du corps humain. C'est un acte psychologique en ce qu'il suppose l'activité volontaire de l'esprit. C'est un acte social en ce qu'il répond à un besoin de communication entre les hommes. Enfin, c'est un fait historique [...]» (1921 : 1-2).

9 Vendryes s'oppose fermement au rapport entre race et langue: «il ne faut pas confondre les caractères ethniques, qui ne peuvent s'acquérir qu'avec le sang, et les institutions - langue, religion, culture - qui sont des biens éminemment transmissibles » (1921 : 276). Que des linguistes tels que Vendryes (et bien d'autres, bien sûr, Saussure entre autres) aient eu à nier de façon péremptoire le rapport entre langue et race montre bien la force d'une telle idée à l'époque. Vendryes, de même, prend ses distances par rapport à l'assimilation fréquente à l'époque entre langue et mentalité : bien qu'on puisse parler, par exemple, d'une mentalité allemande et d'une mentalité française, et qu'on puisse établir qu' «il y a chez [l]es deux peuples une mentalité différente, des goûts, des habitudes, des tempéraments nationaux », il faut penser que " ces tempéraments nationaux ont tout l'air, comme les langues elles-mêmes, d'être des effets et non des causes. Il est aussi arbitraire de faire sortir la langue de la mentalité que la mentalité de la langue. Toutes deux sont le produit des circonstances; ce sont des faits de civilisation » (1921: 277). Et cependant, même si «à quelque partie du langage qu'on s'attache [grammaire, ordre des mots, vocabulaire...], il apparaît qu'on aurait tort d'y voir l'image d'une certaine mentalité », « cela ne veut pas dire qu'il n'y ait aucun rapport entre la mentalité et la langue » (1921:280).

10 Si Vendryes s'efforce de montrer le rapport entre le langage et les conditions où se développe la vie des hommes, il souligne que ce rapport n'est point direct, absolu, mécanique: «les sauvages ne sont pas des primitifs, bien que souvent on leur donne abusivement ce nom. Ils parlent parfois des langues aussi compliquées que les plus compliquées de nos langues; mais il en parlent aussi qui sont d'une simplicité à faire 
envie à nos langues les plus simples » (1921: 7). Les langues des sauvages ne sont point des langues primitives, et certaines d'entre elles peuvent être bien plus complexes que les nôtres (les langues européennes, il s'entend). Ainsi, pour Vendryes, on aurait tort de poser un parallélisme entre degré de civilisation et degré de développement acquis par une langue : les langues des sauvages ne sont point des langues primitives, mais pour autant leur degré de civilisation n'est pas moins arriéré. Henri Berr, auteur de la préface de l'ouvrage, précise que c'est l'homme en dernière instance qui perfectionne la langue : «ce ne peut être la société qui crée les catégories logiques : la société a des besoins, mais elle ne pense pas » (Vendryes 1921 : XVIII).

11 Vendryes pose à la fin de son ouvrage la question cruciale: "s'il n'y a pas dans l'histoire des langues comme un reflet de l'histoire des civilisations» (1921: 413). Il reprend les arguments antérieurs pour montrer qu'il existe un progrès dans l'évolution des langues, qui n'est pas à chercher dans la phonétique, dans la morphologie ou le lexique, mais plutôt dans « la marche du langage vers l'abstraction [qui] est liée à un développement de la civilisation» (1921: 417). Il fonde par là un autre type de différenciation entre langues de peuples civilisés et langues de peuples sauvages : « s'il y a une différence entre les langues des peuples dits civilisés et les langues des sauvages, elle est dans les idées à exprimer plutôt que dans l'expression. Les langues des sauvages peuvent instruire utilement sur les rapports du langage et de la pensée, mais non sur ce qu'a été la forme primitive du langage " (1921: 7). C'est donc la capacité d'abstraction liée à la langue, donc au développement de la pensée, qui diffère entre les unes et les autres, et qui s'inscrit comme facteur de différenciation des langues et des peuples. Aussi, le style petit-nègre, si fréquemment répandu à l'époque du type : « moi y en a manger toi » ou «moi pas peur de lui »-montrait ce préjugé de l'infériorité mentale des peuples colonisés, incapables de penser à la manière européenne. Si certains d'entre eux (ceux qui étaient scolarisés) arrivaient à bien parler une langue, c'est parce qu'ils avaient été « civilisés » et avaient acquis les modes de vie et donc les habitudes mentales des Européens.

Quant à ses positionnements sur la langue française, s'il évite soigneusement de déclarer que c'est la langue française qui permet, le mieux, à la pensée de s'exprimer clairement, il admet aisément les "qualités » traditionnelles qui lui sont octroyées: « les langues modernes comme l'anglais ou le français ont une souplesse, une aisance, une flexibilité extrêmes. Le français se recommande notamment par son exactitude et sa clarté. Bien loin de tolérer les licences, les exagérations, les éclats approuvés par certains langues voisines, il recherche en tout une précision telle qu'il n'admet rien, comme dit Voltaire, qui ait besoin de glose ou d'excuse » (1921: 405). Vendryes apporte finalement un argument essentiel au sujet des qualités de la langue française, considérées par beaucoup comme des qualités intrinsèques (au point qu'on est arrivé à dire qu'«il suffisait de parler français pour être clair»): une langue n'est pas une « entité idéale » qui évolue indépendamment des hommes et poursuit ses fins propres; « les caractères d'une langue se maintiennent tant que ceux qui la parlent conservent les mêmes habitudes de pensée ; ils sont exposés à s'altérer, à s'user, à disparaître [...]. Le langage n'existe pas en dehors de ceux qui pensent et qui parlent [... et] la conscience individuelle n'est qu'un des éléments de la conscience collective qui impose ses lois à chacun" (1921: 420). Dommage qu'on n'ait pas lu Descartes plus attentivement à son époque, car il défendait déjà cette idée si évidente : la langue ne peut point être claire, ni raisonnable, ni précise ; ce sont là des propriétés du discours, donc des locuteurs de la langue, pas de la langue en elle-même: "Ceux qui ont le 
raisonnement le plus fort et digèrent le mieux leurs pensées afin de les rendre claires et intelligibles, peuvent toujours le mieux persuader ce qu'ils proposent, encore qu'ils ne parlassent que bas-breton » (in Vendryes 1921 : 406)

C'est A. Meillet qui va poser, l'un des premiers à notre connaissance $(1918,1928)$, le facteur linguistique comme l'un des problèmes de l'Europe nouvelle qu'on voulait construire au lendemain de la Première Guerre mondiale : ses points de vue montrent cependant une vision traditionnelle, commune à l'époque, qui ne peut se résoudre à accepter la diversité linguistique comme une valeur. Pour lui, la situation linguistique de l'Europe est paradoxale : d'un côté, la civilisation européenne (la science, l'art, les moyens matériels) se renforce et s'unifie, et de l'autre, les langues qui servent d'organe à cette civilisation sont diverses et deviennent constamment plus nombreuses: en effet, de nombreuses langues minoritaires (polonais, hongrois, albanais, estonien, lituanien, etc.) ont accédé au rang de langues nationales, et «chaque nation, si petite soit-elle, veut avoir sa langue de civilisation à elle » $(1928: 1)$ :

$\mathrm{Au}$ fur et à mesure que la civilisation devient plus une, les langues, et même les grandes langues littéraires, sont conduites à exprimer par des procédés matériels différents, mais parallèles, des choses qui sont les mêmes; les notions ne varient pas avec les mots qui les expriment, et, si différentes qu'elles soient par les moyens qu'elles emploient, toutes les langues de l'Europe tendent à être, par ce qu'elles expriment, le calque les unes les autres. (1928:2-3)

Il ne peut non plus accepter de bon gré l'entreprise de "sauvetage artificiel» de langues minoritaires telles que l'irlandais : "À juger des choses en homme civilisé, en Européen, à se placer au point de vue pratique, il est étrange qu'on puisse même proposer à un peuple d'abandonner la grande langue de civilisation, largement ouverte sur le monde, qu'est l'anglais, pour un parler de paysans qui l'emprisonnerait dans un cachot linguistique» (1928: XI). Pour lui, «il y a moins encore d'égalité entre les langues qu'entre les peuples; il n'y a pas de commune mesure entre la valeur intellectuelle et sociale du français et celle du breton et du basque " (1928: XII). Bien sûr, « le bon droit de ceux qui les [ces nouvelles langues] créent ou qui les développent ne peut être contesté » (1928: 3) : cela n'empêche pas qu'il regarde d'un œil désapprobateur ces tendances de la politique linguistique. Il espère cependant que la prise de conscience des "citoyens du monde nouveau qui se crée maintenant " saura "plier la demi-anarchie linguistique d'aujourd'hui à la discipline qu'imposera la civilisation universelle de demain » $(1928: 4)$.

C'est à cette prise de conscience qu'il consacre son livre: il met en relief les inconvénients des langues nationales, et l'abandon progressif des grandes langues de civilisation: "le nationalisme linguistique facilite à la masse un commencement de culture; mais il confine les gens qui parlent une petite langue dans un cercle étroit, d'où ils sortent difficilement » (1928: 238); ou encore : "en se donnant une langue de civilisation propre, une nation s'isole des autres. Elle devient comme une cellule particulière de la civilisation universelle » $(1928: 240)$.

Il montre en revanche les avantages d'une langue commune: "l'unité de langue commune est une force immense pour ceux qui la possèdent » (1928: 244); il donne les exemples de l'espagnol et du portugais en Amérique du Sud; de l'anglais en Amérique du Nord et ailleurs ; c'était le cas du français aux XVIII ${ }^{e}$ et XIX ${ }^{e}$ siècles :

À la fin du XVIII ${ }^{\mathrm{e}}$ siècle, la noblesse russe apprend le français, et, négligeant le russe qui paraissait inculte, la cour de Russie parle dès lors couramment le français, qui a été la langue de l'aristocratie européenne dans la première moitié du XIX ${ }^{\mathrm{e}}$ siècle. $\mathrm{Au}$ 
Levant, le français était la langue usuelle des affaires et de la culture intellectuelle chez tous les sujets de l'ancien empire turc qui avaient des relations avec l'Europe. Les écoles de missionnaires, et plus récemment aussi celles de l'Alliance israélite, ont fait du français la base de leur enseignement des choses occidentales. Il a pu sembler que le français serait pour l'Europe comme une langue commune de civilisation. Le développement s'est arrêté ; le français a cessé de progresser. (1928: 249)

Pour enrayer l'émiettement linguistique de l'Europe, il prône l'emploi « des grandes langues de civilisation comme langues secondes» en Europe (1928: 248); mais l'apprentissage de telles langues ne doit pas se limiter aux aspects pratiques ou «fonctionnels» (pour reprendre un terme actuel) :

Si l'apprentissage des langues étrangères est poussé à fond, de manière à profiter de l'esprit, il demande un temps immense. S'il est superficiel, il n'apporte presque rien à la culture intellectuelle. Tous ceux qui ont passé par de grands hôtels internationaux ont vu des maîtres d'hôtel répondre aisément à des clients qui s'adressent à eux en quatre ou cinq langues différentes; ce que ces hommes ont appris en pratiquant leur métier en divers pays n'a pas vraiment enrichi leur pensée. Pour les gens qui apprennent les langues modernes au point de vue pratique et qui s'intéressent seulement aux formes contemporaines de ces langues, le profit intellectuel de l'apprentissage qu'ils en font est médiocre. (1928: 263)

Il constate que l'« Europe comptera durant bien des années encore beaucoup de petites langues de civilisation », mais il espère que « dès maintenant l'anglais, l'allemand, le français servent en Europe de langues communes de civilisation » $(1928: 286)$.

L'état d'esprit qu'Antoine Meillet manifeste est ainsi révélateur d'une mentalité de l'époque : tout d'abord, la valeur des langues communes, qui sont rehaussées au rang de grandes langues de civilisation:

Une langue parlée sur un petit espace par un petit nombre d'hommes ne peut subsister qu'autant que ceux qui la parlent vivent isolés. On rencontre dans les montagnes du Caucase une quantité de langues distinctes les unes des autres. Les populations qui les emploient ne participent pas à la civilisation de leur temps; dans la mesure où elles ne sont pas bilingues, elles n'entrent en rapport avec le reste de l'humanité que par des interprètes qui connaissent des langues d'usage plus étendu.

Le progrès de la civilisation a donc pour effet d'obliger les gens qui parlent des langues purement locales à connaître en même temps une langue d'usage plus général. (1928: 103)

d'autre part, l'idée du rôle civilisateur des grandes langues :

Dans une région donnée, où se parlent des langues diverses, il n'y a en général qu'une grande langue qui soit la langue de civilisation principale et qui soit en possession d'une sorte de droit à fournir autour d'elle des termes de civilisation. C'est le cas du chinois, par rapport au japonais, au coréen, à l'annamite, ou de l'arabe par rapport au persan, au turc (qui, comme l'hindi, reçoit pourtant des mots de la grande langue de civilisation qu'est, et surtout qu'a été, le persan), au berbère, aux langues des nègres islamisés de l'Afrique. (1928:102)

21 Les réflexions d'Antoine Meillet ont le mérite de placer l'expression «langue de civilisation » dans un contexte moins idéologique (aucun rapport n'y est fait à la race, à une supériorité prétendue de la langue, etc.), et plus culturel, technique, ou scientifique. Il montre par contre une méfiance énorme à l'égard de la diversité linguistique en Europe, et manifeste que seules les grandes langues (anglais, français, allemand) ont le pouvoir (et donc le droit) de jouer un rôle civilisateur : préjugé avec lequel les Européens vont affronter la réalité linguistique de l'Afrique à l'époque. 
Le débat de l'époque sur les rapports entre langue et pensée (psychologie) d'un côté, et de l'autre entre langue et société, connaissait d'autres prises de position intéressantes pour éclairer la complexité de l'expression «langue de civilisation ». On devrait citer les positionnements de bien d'autres linguistes, tels que Ferdinand de Saussure, Ferdinand Brunot, et bien d'autres... Disons simplement que, vivant jusque-là dans l'extase d'une situation privilégiée, la France tarde à se poser la question du rôle du français comme langue internationale dans un contexte politico-économique totalement différent : elle le fera néanmoins après la Seconde Guerre mondiale.

\section{Le français : « langue de civilisation »}

23 Au cours des années 1920 et 1930, l'expression « langue de civilisation » s'est largement installée dans les ouvrages des linguistes: nous avons montré qu'elle révèle une idéologie sur la langue française, mais aussi un positionnement sur la mission civilisatrice de l'entreprise coloniale. On retrouve cette expression après la Seconde Guerre mondiale : elle est reprise dans les propos de nombreux linguistes et dans les discours sur la politique linguistique de l'époque. L'Unesco elle-même s'en fait l'écho. Nous pouvons lire par exemple dans l'Introduction de L'Élaboration du français élémentaire :

Dès 1947, un comité de linguistes où la France était représentée par M. Aurélien Sauvageot, professeur à l'École nationale de langues orientales vivantes, envisageait comme un des moyens les plus efficaces de lutter contre l'ignorance et la misère qui sévissaient en trop de régions du globe, la diffusion de langues de civilisation. Grâce à elles, il serait possible de répandre largement des notions d'hygiène, d'économie domestique, de technique, qui aideraient ces populations «sous-développées " à améliorer leur existence matérielle et à élever leur niveau moral et intellectuel. Deux catégories de langues de civilisation étaient envisagées: d'une part des langues régionales ou locales aménagées en langues de civilisation, d'autre part des langues mondiales (en première ligne le français et l'anglais). (Gougenheim et al. $1956: 7)$

24 André Marie, ministre de l'Éducation nationale français de l'époque, dans la présentation de l'édition du Français élémentaire en 1955 - ouvrage qui répond au vœu de l'Unesco «concernant la diffusion des grandes langues de civilisation »- déclare qu'enseigner le français aux populations indigènes n'était pas seulement une question d'éducation de base (inscrite dans la lutte contre l'analphabétisme), mais fondamentalement un outil d'accès à la " civilisation », la seule civilisation méritant ce nom étant la civilisation occidentale :

De quoi il s'agissait-il à l'origine, sinon d'arracher quelques millions d'hommes dans nos territoires d'Outre-mer à leur isolement spirituel ? [...] Encore ces idiomes [les langues indigènes], si nombreux et si divers, risquent-ils d'enfermer dans la prison du langage les populations qui les pratiquent, que trop souvent n'a pas encore touchées l'école et auxquelles il convient de donner accès à la communauté des hommes en leur fournissant, avec le français, l'instrument indispensable dans le monde moderne à une large et rapide promotion humaine. (Ministère de l'Éducation nationale 1955 : 3-4)

Qu'on ait ressenti le besoin de relever «le niveau moral et intellectuel » des peuples sous-développés ne peut aujourd'hui que surprendre: c'était néanmoins un préjugé commun à l'époque. Quant aux langues de civilisation, elles étaient de deux sortes: d'un côté, les grandes langues régionales ou locales qu'il fallait « aménager en langues 
de civilisation ", entreprise qui fut confiée au Centre des langues locales d'Outre-mer, créé à l'École nationale des langues orientales vivantes (et rien ne fut fait à cet égard) ; de l'autre, les langues mondiales, dont le français et l'anglais, qui n'avaient nullement besoin d'un aménagement quelconque, puisqu'elles étaient de fait des «langues de civilisation ", bien sûr, disons-le, de la civilisation occidentale, considérée comme supérieure aux formes de vie, aux croyances et aux comportements des populations locales.

Le Français élémentaire deviendra ainsi le terrain d'une lutte idéologique extrêmement violente. Avec un curieux paradoxe: ceux qui avaient prétendu renouveler l'enseignement du français à l'aide de cet outil (le ministre, la Commission des linguistes qui est chargée de son élaboration), pour mieux diffuser la "civilisation » occidentale, sont mis sur la sellette sous l'accusation de porter atteinte à la culture française, de laisser les peuples colonisés dans l'ignorance, « de saboter l'enseignement du français dans les régions colonisées" (Marcel Cohen 1955: 74). Les débats permettent toutefois de reprendre une expression qui paraissait oubliée : le français en tant que "langue de culture", qui commence à remplacer l'expression «langue de civilisation » :

Accéder à la culture française [...], c'est apprendre l'histoire du peuple de France, comprendre les caractères du génie national français, faire connaissance avec les traditions des travailleurs de France et avec les œuvres de nos savants, de nos écrivains, de nos artistes. On n'y parviendra que si l'on dispose d'un langage à la fois assez riche et souple pour exprimer l'infinité de faits et d'idées qui constituent ce donné culturel. À cette fonction seule peut être apte notre grande langue nationale, une de celles qu'on appelle précisément langues de culture. (Cohen 1955 : 70)

L'occasion était trop belle pour ne point rappeler que la culture française est liée à des valeurs universelles :

La culture française, dans ses traits les plus essentiels - ceux que lui ont donnés au cours des siècles tant d'écrivains dans les domaines de la poésie, du roman, du théâtre, de la politique, de la science, etc., a incontestablement une valeur émancipatrice.

Avec la langue française authentique, apprise dans les textes, par la lecture et la réflexion en français, ce sont les idées généreuses de justice et d'égalité, l'esprit de libre examen, la critique des idées, l'exemple des révolutions françaises, la mise en cause du régime colonial, qui risquent d'entrer dans la tête des petits élèves d'outre-mer. Or, de cela, le colonialisme ne veut à aucun prix. (Cohen 1955 : 71-72)

À nouveau, l'union « indissoluble » entre langue française et qualités intellectuelles et morales est faite: la langue française «authentique» (pas le français élémentaire, " informe et infirme»), apprise dans les textes, permet d'ouvrir les esprits aux valeurs de liberté, justice, générosité, égalité, libre examen, esprit critique, etc. Qu'une simple question nous soit permise: et si la lecture de ces textes se fait à travers une traduction, l'effet n'est-il plus le même?

Le slogan «à langue pauvre, information réduite, pensée pauvre» (Cohen $1955: 70$ ) s'impose par la simplicité de sa formulation. Aussi, les auteurs chargés de l'élaboration du Français élémentaire seront "obligés » de faire des tractations et d'ajouter à la liste des mots élémentaires - obtenus grâce aux enquêtes de fréquence et de disponibilité de nouveaux "mots permettant d'exprimer des notions morales, civiques, culturelles ", tels que art, courage, effort, justice, liberté, paix, progrès, vérité, etc. Le sacrifice du terme élémentaire (au profit de Français fondamental. Premier Degré, 1959), les assurances données selon lesquelles il ne s'agissait que d'un premier niveau de connaissances et 
qu'il était hors de question de l'appliquer aux programmes de langue maternelle, la composition d'un Français fondamental. Second degré (1959), « fondé essentiellement sur la langue écrite » (Présentation), rassureront les esprits et calmeront les polémiques. Tout rentrait à nouveau dans l'ordre, dans le terrain commun d'entente imposé par les "gardiens de la langue» (Albert Dauzat entre autres) : les mots sont porteurs d'idées; sans les mots correspondants celles-ci n'existeraient pas dans les représentations d'un individu, ou n'existeraient qu'à un état imperceptible ou diffus; restreindre le vocabulaire à apprendre signifie l'impossibilité d'accéder aux idées correspondantes, et donc le maintien de l'intellect dans la simplicité que manifestent les gens incultes ou les peuples primitifs.

30 Ces débats permettent de mettre à jour que les Français continuaient, dans les années cinquante, de posséder un attachement très fort, d'ordre affectif ou émotif, à la langue nationale, réputée claire, subtile et universelle, possédant des vertus émancipatrices à elle seule. Ce qu'on remarque moins souvent c'est que cette représentation de la langue, comme le met en relief Henri Besse, a été accompagnée d'un revers de médaille, d'une "histoire obscure, brutale et chauvine » faite de despotisme linguistique, de maintien du "corset de la grammaire", de «vigilance sourcilleuse des écarts vernaculaires " ou d'opposition aux tentatives très rationnelles de simplification de l'orthographe (Besse 1979 : 23).

\section{En guise de conclusion}

L'expression «langue de civilisation » cesse d'être utilisée dans les années 1960 : le processus de décolonisation entrepris par le général De Gaulle la rendait désuète et incommode, car elle renvoyait à une façon de concevoir les anciennes colonies dans le rapport hiérarchisé antérieur : «La France, contrainte de renoncer à ses colonies, était amenée à maintenir son rayonnement par d'autres liens que ceux d'un empire dominé par les intérêts de la métropole " (Argaud 2006 : 26). Et cette évolution dans les esprits se produit précisément plus vite chez les enseignants, parfois plus sensibles à l'anticolonialisme que d'autres secteurs de la société, et plus lucides sur les risques de voir se maintenir les mêmes liens à travers les nouveaux accords de coopération " (Argaud $2006: 27$ ). Malgré tout, la politique éducative et culturelle postcoloniale est "pleine de paradoxes et d'ambiguïtés" (ibid. 27). Certains continuaient de voir ainsi dans la défense du français en tant que langue internationale le besoin de "l'assimilation des modes de pensée française ", considérant que "seul l'usage répandu $\mathrm{du}$ français pourrait empêcher l'aliénation culturelle de nations menacées par l'hégémonie de l'anglais » (Françoise de la Serre, in Argaud 2006 : 27).

Les méthodes issues du CRÉDIF (Centre de recherche et d'études pour la diffusion du français)ou du BEL (Bureau d'étude et de liaison pour l'enseignement du français dans le monde), institutions créées toutes deux en 1959, cherchaient avant tout à assurer la maîtrise du français parlé courant. Le "français langue étrangère" (FLE) était né comme champ disciplinaire (Coste 1987 : 30). Si la civilisation y est concernée (le BEL devient en 1966 le BELC: Bureau pour l'étude de la langue et de la civilisation françaises) et constitue une composante essentielle du FLE, elle l'est dans le sens anthropologique (les modes de vie) et dans le sens de "culture", les deux termes se rejoignant à tel point qu'É. Argaud, dans son étude de la revue Le Français dans le monde (1961-1976), soutient cette idée, qui montre le fossé qui s'est creusé par rapport à 
l'idéologie antérieure : «le refus d'une conception obsolète de la civilisation conduit les spécialistes du FLE à établir une équivalence entre civilisation et culture, qui deviennent ainsi des synonymes, et à mettre en avant l'approche anthropologique ». Elle affirme ainsi que «le champ du FLE ne reconnaît pas de façon explicite, dans les années 1960-70, le rôle légitimant qu'a eu civilisation dans l'entreprise coloniale " (2006:7-8).

La notion de civilisation a donc fortement changé et, de ce fait, un nouveau domaine de réflexion et d'études se crée, autant dans des approches théoriques que pratiques (ou méthodologiques): celui de la "civilisation française», en tant que composante essentielle de l'enseignement du FLE. Dans son relevé des emplois du terme civilisation, Évelyne Argaud signale qu'il est utilisé au sein de trois types de discours (qui se recoupent souvent) : la politique culturelle de la France; l'approche sociologique ou anthropologique, l'approche pédagogique. "Parfois mais rarement, il introduit la notion de jugement de valeur et de hiérarchie : 'civilisation supérieure'« (2006:18); une page semble avoir été tournée. Pas par tout le monde cependant, car l'emprise des notions antérieures au sujet de la langue et de la culture françaises se maintient chez certains linguistes et politiciens. Ainsi, les organisateurs du VIII Congrès quadriennal de l'Association des professeurs de français d'Afrique et de l'Océan indien sur le thème "Le français, une langue partenaire pour le développement de l'Afrique", adoptent une série de résolutions, dont la quatrième dit: "changer de discours sur la langue française: plus de discours sur les vertus civilisatrices de la langue et de la culture françaises ", et la cinquième : "éviter de chanter les vertus de la langue maternelle à un peuple qui a déjà été francisé ». Et c'est en 2007 (du 24 au 27 septembre) que s'est tenu ce Congrès !

\section{BIBLIOGRAPHIE}

ARGAUD, Évelyne (2006). La civilisation et ses représentations : étude d'une revue, le Français dans le monde (1961-1976), Berne, Peter Lang.

BERGOUNIOUX, Gabriel (1989). «Le francien (1815-1914) : la linguistique au service de la patrie », Mots, 19, 23-39.

BESSE, Henri (1979). « Contribution à l'histoire du français fondamental », Le Français dans le monde, 148, 23-30.

BOURDIEU, Pierre (2001). Langage et pouvoir symbolique, Paris, Fayard.

CALVET, Louis-Jean (1974). Linguistique et colonialisme. Petit traité de glottophagie, Paris, Payot.

- (1987). La guerre des langues et les politiques linguistiques, Paris, Payot.

COHEN, Marcel et al. (1955). Français élémentaire? Non, Paris, Éditions Sociales.

COSTE, Daniel (1987). Institution du FLE et implications de la linguistique appliquée, Thèse d'État, Paris VIII, Sciences du langage. 
FUMAROLI, Marc (1984). «L'apologétique de la langue française classique », Rhetorica, $2: 2$, 139-161.

GOUGENHEIM, Georges et al. (1956). L'Élaboration du français élémentaire, Paris, Didier.

HÉNON, M. (1938). Lectures historiques, Livre du maître, t. II. Paris, Nathan.

Ministère de l'Éducation Nationale (1955). Le Français élémentaire,Paris, Publications de l'Éducation Nationale.

- (1959). Le Français fondamental. Premier Degré, Paris, Institut Pédagogique National.

- (1959). Le Français fondamental. Second degré, Paris, Institut Pédagogique National.

MEILLET, Antoine (1928). Les langues dans l'Europe nouvelle, Paris, Payot. Cette édition comporte une série de modifications par rapport au texte de la première édition (1918) et y ajoute un appendice de Lucien Tesnière.

OLIVIERI, Claude (1984). Vingt-cinq ans dans l'évolution d'une politique de diffusion du français langue étrangère. 1945-1970, Colloques du Centenaire, École Normale Supérieure de Saint-Cloud.

PETROFF, André-Jean et ENGLER, Rudolf (2004). Saussure : la langue, l'ordre et le désordre, Paris, L'Harmattan.

PROST, Antoine (1968). Histoire de l'enseignement en France (1800-1967), Paris, A. Colin.

RAMBAUD, Alfred (1911). Histoire de la civilisation française, t. II, Paris, A. Colin.

VENDRYES, Joseph (1921). Le langage. Introduction linguistique à l'histoire, Paris, La Renaissance du livre.

\section{NOTES}

1. Le terme « civilisation » apparaît pour la première fois dans un texte de Mirabeau de 1756, et a eu une rapide diffusion, comme le signale Claude Liauzu : « le succès de la notion tient à ce qu'elle rend compte d'une nouvelle philosophie de la nature et de l'homme, fondée sur l'idée du progrès, qu'elle résume l'esprit des Lumières tout entier» (in Argaud 2006 : 5).

\section{RÉSUMÉS}

Notre réflexion s'organise en deux volets qui se succèdent d'un point de vue chronologique : le premier veut montrer la genèse de l'expression « langue de civilisation ", qui fait fortune dans les années 1920,30, 40 et 50, et les notions (multiples et complexes) auxquelles elle est rattachée, à travers les positionnements d'une série de linguistes du début du $\mathrm{XX}^{\mathrm{e}}$ siècle ; une fois construite cette notion, et acceptée par la communauté des «intellectuels» de l'époque, elle est reprise pour appuyer une action politico-linguistique concrète, axée sur l'idée du français comme langue de civilisation (Unesco, Français élémentaire). Le tournant pris par la décolonisation écartera rapidement cette expression au profit de deux autres: le français «langue de culture » et « langue et civilisation françaises ». 
Our reflection is organized in two parts, one leads to the other from a chronological point of view. The first one wants to show the genesis of the expression "language of civilization", developed in the years 1920-50, and the notions (multiple and complex) to which it relates. To achieve it we analyse the points of view of a series of linguists of this period. Once this notion is constructed, and accepted by the community of the "intellectual ones" of this time, it is used to support a political-linguistic action based on the idea of French as a language of civilization (Unesco, elementary French). The decolonization will rapidly drop this expression in favour of two other: French language as "language of culture" and "French language and French civilization".

\section{INDEX}

Mots-clés : langue de civilisation, langue de culture, français élémentaire, idéologie de la langue, préjugé linguistique, Joseph Vendryes, Antoine Meillet, Marcel Cohen, UNESCO

Keywords : Language of civilization, language of culture, elementary French, language ideology, linguistic prejudice, Joseph Vendryes, Antoine Meillet, Marcel Cohen, UNESCO

\section{AUTEURS}

\section{JAVIER SUSO LÓPEZ}

Université de Grenade, Espagne

jsuso@ugr.es

\section{MARIA EUGENIA FERNÁNDEZ FRAILE}

Université de Grenade, Espagne.

mefraile@ugr.es 